\title{
One Field Epidemiologist per 200,000 Population: Lessons Learned from Implementing a Global Public Health Workforce Target
}

Seymour G. Williams, Robert E. Fontaine, Reina M. Turcios Ruiz, Henry Walke, Kashef Ijaz, and Henry C. Baggett

The World Health Organization monitoring and evaluation framework for the International Health Regulations (IHR, 2005) describes the targets for the Joint External Evaluation (JEE) indicators. For workforce development, the JEE defines the optimal target for attaining and complying with the IHR (2005) as 1 trained field epidemiologist (or equivalent) per 200,000 population. We explain the derivation and use of the current field epidemiology workforce development target and identify the limitations and lessons learned in applying it to various countries' public health systems. This article also proposes a way forward for improvements and implementation of this workforce development target.

Keywords: Field epidemiology, Workforce development, Epidemic management/response, Global health security

$T^{n}$ he World Health Organization's (WHO) Joint External Evaluation (JEE) tool, part of the International Health Regulations (IHR, 2005) monitoring and evaluation framework, states, regarding workforce development needs, that an "optimal target for surveillance is one trained field epidemiologist (or equivalent) per 200,000 population. ..." This workforce development target provides a measurable standard for IHR (2005) human resource compliance and preparedness by countries. ${ }^{2}$ The target also supports monitoring and motivation for field epidemiology workforce capacity development. This target can forecast staffing adequacy and skill gaps, especially in planning for public health events and emergencies. ${ }^{2-4}$ At the country level, this target facilitates workforce development planning to ensure that the variety of public health functions are fulfilled (Figure 1).

The Field Epidemiology Training Programs (FETPs) have been the primary way for countries to systematically produce field epidemiologists. FETPs are competencybased in-service training programs that have helped more than 65 countries strengthen surveillance and response capacities. ${ }^{5}$ Field epidemiologists investigate the occurrence

Seymour G. Williams, MD, is Team Lead, Field Epidemiology Training Program; Robert E. Fontaine, MD, is Senior Advisor, Field Epidemiology Training Program; Reina M. Turcios Ruiz, MD, is Team Lead, Monitoring and Evaluation; all in the Workforce and Institute Development Branch, Division of Global Health Protection, Center for Global Health, US Centers for Disease Control and Prevention (CDC), Atlanta, GA. Henry Walke, MD, is Director, Division of Preparedness and Emerging Infections, National Center for Emerging and Zoonotic Infectious Diseases, CDC, Atlanta, GA. Kashef Ijaz, MD, is Principal Deputy Director, and Henry C. Baggett, MD, is Chief, Workforce and Institute Development Branch; both in the Division of Global Health Protection, Center for Global Health, CDC, Atlanta, GA. Dr. Williams and Dr. Baggett are senior co-authors. The findings and conclusions in this report are those of the authors and do not necessarily represent the official position of the US Centers for Disease Control and Prevention. 
Figure 1. Public Health Functions Needing an Epidemiologic Workforce

\begin{tabular}{|l|}
\hline $\begin{array}{l}\text { Operating surveillance } \\
\text { Designing data systems } \\
\text { Analyzing incoming data } \\
\text { Evaluating data collection, quality, and analysis and utility } \\
\text { Responding to public health emergencies } \\
\text { Infectious disease outbreaks } \\
\text { Noninfectious disease outbreaks } \\
\text { Environmental threats } \\
\text { Natural and man-made disasters } \\
\text { Preparing for cross-border threats } \\
\text { Supporting control and prevention programs } \\
\text { Infectious diseases (including vaccine preventable, tuberculosis, HIV) } \\
\text { Chronic diseases } \\
\text { Maternal and child health } \\
\text { Injuries } \\
\text { Healthcare-associated problems (healthcare quality) } \\
\text { Environmental health } \\
\text { Occupational health }\end{array}$ \\
\end{tabular}

of diseases and other health-related conditions or events in defined populations, actively responding to control urgent public health problems in the community. ${ }^{6,7}$ FETP participants learn the profession of epidemiology through service by conducting routine surveillance and addressing priority public health problems, including responding to outbreaks, emergencies, and humanitarian crises. For example, the Nigerian FETP responded quickly and stopped an outbreak of Ebola in the densely populated city of Lagos, thereby preventing Ebola from gaining a foothold there and spreading further. ${ }^{8}$ The Brazilian FETP investigated a measles outbreak linked to importation due to Venezuelan migration. ${ }^{9}$ Having an FETP or other applied epidemiology program in place is one of the JEE human resources indicators. ${ }^{1}$ Countries and their supporting partners use FETPs to build field epidemiology capacity and have applied the 1 per 200,000 field epidemiology target to determine workforce gaps and investment needs and guide training strategies. 5,10

Given our experience implementing FETP in selected countries using the current field epidemiology workforce development target, this article summarizes how the target was developed, how it has been used, and what has been learned, and proposes a way forward.

\section{How Was the Target Developed?}

Estimates of epidemiology workforce capacity have been regularly published by the US Council of State and Territorial Epidemiologists (CSTE). The CSTE conducted 5 Epidemiologic Capacity Assessment (ECA) surveys from 2001 to $2017 .{ }^{11-15}$ As part of these surveys, the CSTE asked all state epidemiologists in the 50 states plus the District of Columbia, the US territories, and the federated states of Micronesia about the current numbers of and needs for additional epidemiologists to reach an optimal level. They asked state epidemiologists to query their public health units to estimate the actual and optimal epidemiologic staffing in 8 public health program areas and in 4 essential services for public health. They defined an epidemiologist according to Last's Dictionary of Epidemiology ${ }^{7}$ and included all staff functioning as epidemiologists, regardless of professional training.

These surveys showed an optimal level from 1.1 to 1.4 epidemiologists per 100,000 population for the state level. The national and local level epidemiology staff were not included in the ECA (only epidemiologists at the state levels). An acceptable level of 1:100,000 was proposed for US state health departments to "gauge" their "epidemiology capacity" and have a common standard to "roughly assess the adequacy of their epidemiology workforce."16

Between 2010 and 2012, while developing a proposal for strengthening field epidemiology capacity internationally, FETP experts at US CDC concluded that a similar population-based metric could serve as a national target for the field epidemiology workforce for countries outside the United States. This metric would help countries grossly estimate the minimal adequacy of their field epidemiology workforce, focus training resources, help donors quantify workforce needs, and help countries progress toward a globally endorsed target. Accordingly, the experts to account for workforce-related and economic challenges in low-resource countries readjusted from the US-based 1:100,000 target and proposed a 1:200,000 target. However, because there were no additional international ECA-like data available 
to inform development and use of this target, the experts decided to use FETP graduates as a proxy for epidemiologists; graduates from 2-year (advanced) and the 9-month (intermediate) FETPs would count toward this target, based on the standard competencies of these programs, ${ }^{5,17}$ whereas graduates from the 3-month (Frontline) FETP would not count. ${ }^{18}$ FETP Frontline tier training develops the skills for disease surveillance officers at district levels and below, while intermediate and advanced training develop field epidemiology skills for staff at the regional and national levels of a country's public health system.

\section{How Has the Target Been Used?}

Both the Global Health Security Agenda (GHSA) in 2014 and the JEE in 2015 incorporated the 1 per 200,000 target as a human resource indicator of sufficient field epidemiology capacity. ${ }^{1,4}$ This target is being used to track the implementation and impact of investments in FETPs and other applied epidemiology training programs. ${ }^{5}$ Individual country FETPs have used the target to determine when they have trained sufficient numbers to reach an adequately sized epidemiologic workforce. ${ }^{10}$

Among the $17 \mathrm{CDC}$ partner countries receiving US CDC support for FETP, 3 have reached the 1:200,000 target based on counting the number of staff completing advanced and intermediate FETP (Figure 2). ${ }^{19}$ The count of FETP-trained epidemiologists in the larger GHSA countries was similar to the number trained in the smaller countries, despite the much higher target. In response, some of these larger countries have increased their FETP class size by a factor of 3 or 4 and launched subnational intermediate-level FETPs and training sites.

\section{What Have We Learned?}

The key lesson from using the target is that it helps countries, donors, and implementers decide on which actions should take priority in order to increase target attainment. The current practice of placing 1 resident advisor (a senior epidemiologist assigned to the country by US CDC to support the growth and development of the FETP) in a country to train 5 to 15 persons per year is too slow to meet the epidemiology workforce needs for large countries in the near term. Program implementers will need to plan from the beginning on how to quickly ramp up training after the initial 1 to 2 years of a FETP to build local leadership capacity to run these FETP independently of resident advisors, and to track the progress in attaining the 1:200,000 target for concurrently meeting the field epidemiology needs of the country's public health system.

Having a measurable target for field epidemiology workforce capacity has proven valuable to accelerate progress toward meeting IHR (2005) human resource capac- ity requirements, but there are inherent limitations in a single national target derived from state-based capacity assessments in the United States. First, the current metric does not include a definition of a field epidemiologist. Most countries' health ministries do not use a standard definition of a field epidemiologist or include an epidemiologist job classification in their human resources administrative structure. The lack of a standard, functional definition of field epidemiologist limits precise measurement and application of the target and challenges assessment of the target for an individual country or across countries.

Using FETP graduates as a proxy for counting field epidemiologists does not work for countries that have no FETP and misses epidemiologists that have become competent through experience in countries that do have a FETP. Furthermore, a single, national measure of field epidemiologists does not consider different epidemiologic competencies at different levels of the public health system. For example, local or district health offices need staff with skills in surveillance, data quality, case investigation, and outbreak detection but might not need staff with all of the field epidemiology competencies developed in a 2-year course.

Second, the current target does not consider geographic distribution of field epidemiologists. Epidemiology expertise often clusters in major metropolitan areas, leaving rural areas uncovered and vulnerable. Even geographic distribution of field epidemiologists might be especially relevant in countries with very large populations (eg, China, India, Nigeria), where prioritizing the achievement of a baseline minimal geographic coverage in subnational units could happen faster and would have a greater impact than merely aiming for a single national target that could take decades to reach (Figure 1). In less populous countries, achieving a single national target could be relatively easy but could create a false sense of security if key subregions or programs lack epidemiology capacity.

Further complicating the full adoption of national field epidemiology workforce targets is that global health workforce initiatives focus primarily on the health services delivery workforce and do not recognize epidemiologists. The WHO's Global Strategy on Human Resources for Health: Workforce 2030 and the High-level Commission on Health Employment and Economic Growth Action Plan call for National Health Workforce Accounts (NHWA) "to create a harmonized, integrated approach for annual and timely collection of health workforce information ... and define core indicators in support of strategic workforce planning and global monitoring." ${ }^{20,21}$ However, while the NHWA handbook references the need for an applied epidemiology workforce using language consistent with the JEE, it does not provide guidance on counting that workforce. The NHWA uses the International Labor Organization's International Standard Classification of Occupations, which does not include epidemiologists or most other public health occupations. ${ }^{22}$ Therefore, this global health 

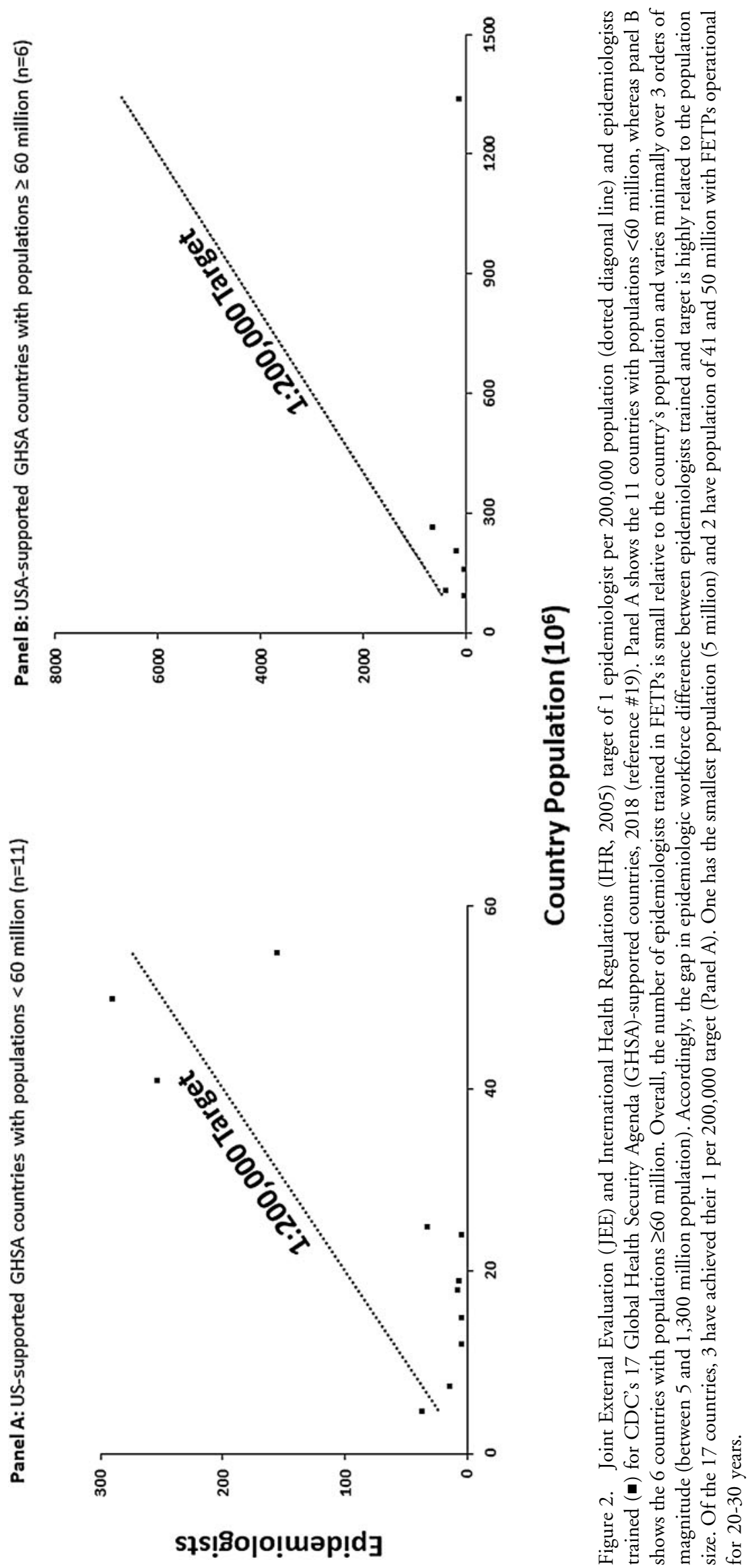
workforce initiative does not offer guidance on whom to count as providing epidemiologic services in a country.

\section{What Is a Possible Way Forward?}

All country stakeholders should be reminded of the value of this field epidemiology workforce target to measure progress toward improving global health security. At the same time, they should also be made aware that specific country-level data (eg, geographic distribution and competencies of epidemiologists) are needed to refine the target to meet a country's epidemiology workforce needs. As part of public health resource planning, countries will need to identify existing information about their public health workforce, availability and adequacy of field epidemiology training, and administrative data to derive country-specific estimates of field epidemiology needs. These estimates will require tailoring to, for example, the administrative structure of the country, the country's disease control programs (eg, for tuberculosis, malaria, HIV, noncommunicable diseases, injuries, etc), the disease burden profile, the healthcare system, the existing epidemiologic workforce, and the potential pool of people with enough scientific and mathematical aptitude to be trained as epidemiologists. The workforce data should be regularly reviewed not only to assess the attainment of targets but to identify opportunities for refinement to serve the public health priorities of the country.

WHO and other multinational public health workforce organizations that support FETPs worldwide should convene relevant stakeholders, including country representatives, regional public health organizations, and other funding institutions, to discuss attaining the field epidemiology workforce targets, develop guidance to improve those targets, and plan to build the evidence base to inform future adjustments. Consistent with that recommendation, the Global Field Epidemiology Roadmap, a product of a meeting of FETP leaders and partners from across the globe, calls for applied epidemiology workforce targets at each level of a country's public health system to achieve the IHR (2005) global health security capacities. ${ }^{23}$ These stakeholder meetings ought to remain closely linked to and be advanced under the umbrella of the IHR (2005) monitoring and evaluation framework, including the JEE, GHSA 2024, and other global and regional health security initiatives. $^{24}$ The JEE human resources indicators call for all countries to develop a multisectoral health workforce strategy, which should include the field epidemiology workforce. However, guidance is needed to develop these strategies. Health sector labor market analyses, recognized as critical to the Human Resource for Health Strategy 2030, should also include the public health workforce and have position descriptions for epidemiologists at the different levels of the government public health system. ${ }^{20}$

In conclusion, targets and indicators, such as the 1 field epidemiologist per 200,000 population, are important not only to measure progress but also to identify challenges that prevent progress, so that partners and stakeholders can work together to find solutions. Every target and indicator (and metric) has its limitations. However, partners should regularly evaluate and, when appropriate, revise the indicator and/or target to better maximize their usefulness for local and global settings. Having a measurable target is better than not having one at all. The targets can be improved or refined, but the perfect should not be the enemy of the good.

\section{REFERENCES}

1. World Health Organization. Joint External Evaluation Tool. International Health Regulations (2005). 2d ed. Geneva: WHO; 2018. https://apps.who.int/iris/bitstream/handle/10665/259961/ 9789241550222-eng.pdf?sequence $=18$ isAllowed $=y$. Accessed December 10, 2019.

2. Ijaz K, Kasowski E, Arthur RR, Angulo FJ, Dowell SF. International Health Regulations-what gets measured gets done. Emerg Infect Dis 2012;18(7):1054-1057.

3. Global Health Security-Vision and Overarching Target. Undated. https://www.cdc.gov/globalhealth/healthprotection/ pdf/ghs_overarching_target.pdf. Accessed December 10, 2019.

4. US Centers for Disease Control and Prevention. Global Health Secuirty Agenda: GHSA Workforce Development Action Package GHSA Action Package Detect-5). December 24, 2014. https://www.cdc.gov/globalhealth/security/actionpack ages/workforce_development.htm?CDC_AA_refVal=https\% $3 \mathrm{~A} \% 2 \mathrm{~F} \% 2 \mathrm{Fwww} . c d c . g o v \% 2 \mathrm{Fglobalhealth} \% 2 \mathrm{Fsecurity} \% 2 \mathrm{~F}$ workforce_development.htm. Accessed December 10, 2019.

5. Jones DS, Dicker RC, Fontaine RE, et al. Building global epidemiology and response capacity with field epidemiology training programs. Emerg Infect Dis 2017;23(13):158-165.

6. Koo D, Thacker SB. In Snow's footsteps: commentary on shoe-leather and applied epidemiology. Am J Epidemiol 2010;172(6):737-739.

7. Last J, ed. A Dictionary of Epidemiology. 4th ed. New York: Oxford University Press; 2001.

8. Marston BJ, Dokubo EK, van Steelandt A, et al. Ebola response impact on public health programs, West Africa, 2014-2017. Emerg Infect Dis 2017;23(Suppl):S25-S32.

9. Elidio GA, França GVA, Pacheco FC, et al. Measles outbreak: preliminary report on a case series of the first 8,070 suspected cases, Manaus, Amazonas state, Brazil, February to November 2018. Euro Surveill 2019;24(2).

10. Reddy C, Kuonza L, Ngobeni H, Mayet NT, Doyle TJ, Williams S. South Africa field epidemiology training program: developing and building applied epidemiology capacity, 2007-2016. BMC Public Health 2019;19(Suppl 3):469.

11. Arrazola J, Binkin N, Israel M, et al. Assessment of epidemiology capacity in state health departments-United States, 2017. MMWR Morb Mortal Wkly Rep 2018;67(33):935-939.

12. Centers for Disease Control and Prevention. Assessment of the epidemiologic capacity in state and territorial health departments-United States, 2001. MMWR Morb Mortal Wkly Rep 2003;52(43):1049-1051.

13. Centers for Disease Control and Prevention. Assessment of epidemiology capacity in state health departments-United 
States, 2009. MMWR Morb Mortal Wkly Rep 2009;58(49): 1373-1377.

14. Hadler JL, Lampkins R, Lemmings J, et al. Assessment of epidemiology capacity in state health departments-United States, 2013. MMWR Morb Mortal Wkly Rep 2015;64(14): 394-398.

15. Centers for Disease Control and Prevention. Assessment of epidemiologic capacity in state and territorial health departments-United States, 2004. MMWR Morb Mortal Wkly Rep 2005;54(18):457-459.

16. Boulton ML, Lemmings J, Beck AJ. Assessment of epidemiology capacity in state health departments, 2001-2006. J Public Health Manag Pract 2009;15(4):328-336.

17. Lopez A, Caceres V. Central America Field Epidemiology Training Program (CA FETP): a pathway to sustainable public health capacity development. Hum Resour Health 2008;6:27.

18. Andre AM, Lopez A, Perkins S, et al. Frontline Field Epidemiology Training Programs as a strategy to improve disease surveillance and response. Emerg Infect Dis 2017; 23(Suppl):S166-S173.

19. Tappero JW, Cassell CH, Bunnell BE, et al.; Global Health Security Science Group. US Centers for Disease Control and Prevention and its partners' contributions to global health security. Emerg Infect Dis 2017;23(Suppl):S5-S14.

20. World Health Organization. Global Strategy on Human Resources for Health: Workforce 2030. Geneva: WHO; 2016. https:// apps.who.int/iris/bitstream/handle/10665/250368/97892415 11131-eng.pdf?sequence=1. Accessed December 10, 2019.

21. World Health Organization. National Health Workforce Accounts: A Handbook. Geneva: WHO; 2017. https://apps. who.int/iris/bitstream/handle/10665/259360/9789241513 111-eng.pdf?sequence=1. Accessed December 10, 2019.

22. Internationl Labour Organization. International Standard Classification of Occupations. June 9, 2010. https://www.ilo. org/public/english/bureau/stat/isco/. Accessed December 10, 2019.

23. TEPHINET. The Global Field Epidemiology Roadmap. 1997. https://www.tephinet.org/the-global-field-epidemiologyroadmap. Accessed December 10, 2019.

24. Global Health Security Agenda (GHSA) 2024 framework. November 2018. https:/ghsa2024.files.wordpress.com/ 2019/11/ghsa-2024-framework.pdf. Accessed December 10, 2019.

\author{
Address correspondence to: \\ Seymour Williams, MD, MPH \\ Division of Global Health Protection \\ Center for Global Health \\ Centers for Disease Control and Prevention \\ 1600 Clifton Road, MS: E-98 \\ Atlanta, GA 30329 \\ Email: Sjw9@cdc.gov
}

